\title{
Airway Management of Covid 19 and Lowcost Ventilators-Aboonor Bane?
}

\author{
Shikha Singh ${ }^{1}$, Rakesh Kumar Jha ${ }^{2}$ and Dhruba Hari Chandi ${ }^{3}$ \\ ${ }^{1}$ FirstYear MBBSStudent,Datta Meghe Medical College, Shalinitai \\ Meghe Hospital and Research Centre, Nagpur, India \\ ${ }^{2}$ Tutor Department. of Biochemistry Datta Meghe Medical College, Shalinitai \\ Meghe Hospital and Research Centre Nagpur, India \\ ${ }^{3}$ Department of Microbiology Jawaharlal Nehru Medical College, Datta Meghe \\ Institute of Medical Sciences Sawangi (Meghe), Wardha \\ Corresponding author email: jhaarakesh1993@gmail.com
}

\section{ABSTRACT}

Corona viruses are a wide group of pathogens that affect both humans and animals. It spreads in humans by human-to-human interaction, as seen with MERS and SARS. Close contact allows the virus to spread by droplets from coughs, sneezes, and conversations. The incubation period varies from 2 to 14 days, and it is still unknown in many cases. Mild symptoms such as sore throat, headache, cough, and malaise are treated with antipyretics and antitussives, as well as nutritive treatment, depending on the symptoms.Oxygen therapy, anticoagulation, and corticosteroids are given with antiviral drugs for mild symptoms. The demand for supply must be maintained. In extreme cases, drug treatment should be continued and oxygenation maintained early in the disease; but, as the disease progresses to ARDS, a non-invasive technique providing oxygen via a mask or nasal cannula and preserving $\mathrm{SpO} 2$ and $\mathrm{FiO} 2$ is used first. If the condition worsens to the point that the Lung no longer functions, a compliant endotracheal intubation is performed in order to administer artificial ventilation in an environment where expertise is available. Patients with septic shock are started on antimicrobial treatment, fluid loadings are maintained, and vasopressors are administered. Glucocorticoids can be used for a limited time to treat a patient's deteriorating condition. Airway Management of COVID19 and LowCost Ventilators-aboonor bane? As a result, disease prevention at the earliest stage is considered a condemnatory measure. However, one must be prepared for any mishaps in the manifestation of disease as it progresses to complication. Invasive procedures such as mechanical ventilation and non-invasive procedures such as nasal cannula oxygenation must be used successfully, depending on the patient's capacity for reaction to the care offered. Given the unpredictability of the future in relation to the disease, it would be advantageous if the country were to be equipped in advance with manufactured low-cost ventilators.

\section{KEY WORDS: AIRWAY MANAGEMENT, SYMPTOMATIC TREATMENT,COVID- 19,MECHANICAL VENTILATION, LOW COST VENTILATOR.}

\section{INTRODUCTION}

Since 12thDecember 2019, an outbreak of unexplained acute respiratory tract infection has been recorded in Wuhan, China, most likely related to a seafood market.

Biosc Biotech Res Comm P-ISSN: 0974-6455 E-ISSN: 2321-4007

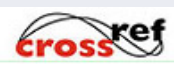

Identifiers and Pagination

Year: 2021 Vol: 14 No (6) Special Issue

Pages: 267-270

This is an open access article under Creative

Commons License Attribn 4.0 Intl (CC-BY).

DOI: $h t t p: / / d x$.doi.org/10.21786/bbrc/14.6.56
Bats may be the potential SARS corona virus 2 reservoirs, according to several studies. Despite this, there is no evidence that SARS corona virus 2 originated in the seafood industry. Bats, on the other hand, are a source of a wide variety of CoVs, as well as viruses such as SARS Corona Virus and MERS Corona Virus.According to virus genome sequencing, COVID-19 was compared to Bat CoV RaTG13 in the genome and showed a 96.2 percent overall genome sequence identity, suggesting that bat $\mathrm{CoV}$ and human SARS corona virus 2 may have the same source, despite the fact that bats are not available for sale on this seafood bazaar. Furthermore, similarity of protein sequences and phylogenetic analysis revealed

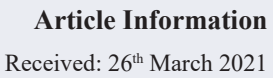

ccepted after revision: $28^{\text {th }}$ May 2021 
identical receptor residues in most species, implying that another midway source, such as tortoises and snakes, is more likely (Rakesh Kumar Jha, et al 2020).

Symptom of Covid-19: Using a sample of 1099 laboratory-certified cases, a recent study led by Prof. Nan-Shan Zhong found that the standard clinical expression included high temperature, cough, and tiredness (88.7\%), (67.8\%), and (38.1\%), respectively, as well as sputum production in 33.4 percent, breathing difficulties in $18.6 \%$, sore throat in 13.9 percent, and headache in 12\%. GIT signs included loose motion (3.8 percent) and vomiting in some patients (5.0 percent) (Lu H, et al 2020). Clinical manifestations in Hubei province were consistent with 41,99, and 138 patients in previous studies.Fever and cough were the most common symptoms, while upper respiratory symptoms and GIT symptoms were rare, suggesting differences in viral tropism from SARS Corona Virus, MERS CoV, and flu. Elderly people and those with underlying disorders (such as hypertension, COPD, diabetes, and CVD) developed ARDS, septic shock, metabolic acidosis, and prolonged coagulation deficiency, which sometimes resulted in death.

What Is Mechanical Ventilation: When a patient is in desperate need, during any surgical operation or serious illness, it is used to support or replace spontaneous breathing with the aid of physical instruments or machines. When there is a need for protection against aspiration in the airway, when the patient is suffering from hypoxic respiratory failure and requires a high dose of inspired oxygen, when there is an obstruction in the upper airway, and when there is hypercapnic respiratory failure requiring assisted ventilation, the patient is put on venti (Stephanie M. Sison et al 2021).

\section{Types of mechanical ventilation}

- The non-invasive technique where there's no insertion of any tube in the air passage of the patient whose.

- The invasive procedure comprising ventilation of patient by invading the trachea with a tube insertion and then connecting it to the ventilator usually come into play for lungs that are weaker, ARDS lungs or neuromuscular weakness causing nonfunctional chest wall. (Javed, n.d.)

Management of ARDS and Hypoxemic respiratory failure (Lentz S, et al 2020): When a patient does not respond to regular oxygen therapy, he or she may need more work to breathe, which is provided by a face mask with a reservoir bag. For lung protection ventilation, a protocol has been established:

Goal Sp02- 88 to 95 percent and/or Pa02- 55 to 80 $\mathrm{mmHg}$, tidal volume $6 \mathrm{ml} / \mathrm{kg}$, RR-16-35/min, PEEP-5-15 cm H2O, target Sp02- 88 to 95 percent and/or PaO2- 55 to $80 \mathrm{mmHg}$.

Non-Invasive Mechanical Ventilation Or High Flow Nasal Cannula Oxygen (HFNO): It aids in the reduction of intubation use. HFNO should not be given to patients with hypercapnia, hemodynamic dysfunction, multiorgan failure, or irregular mental state, but it may be given to patients with mild and moderate non-invasive hypercapnia. In the event of an emergency, the patient must be monitored for at least 1 hour by trained staff who are capable of ET intubation (Naqvi, Waqar M., and ArtiSahu. 2020) Non-invasive ventilation: PS 5-15 $\mathrm{cmH} 20$ adjusted to $5-7 \mathrm{ml} / \mathrm{kg}$ tidal volume, PEEP $5-10 \mathrm{~cm}$ $\mathrm{H} 2 \mathrm{O}$, FiO2 0.5-1.0 titrated to goal Sp02> 94 percent.

Points to be Remembered: Expert should do the ET intubation under the cautious airborne terms. Children, pregnant or obese patients may get desaturated easily during intubation. To avoid such consequences, one must pre-oxygenate with face mask with reservoir bag at 100\% FiO2 for 5 minutes with face mask with reservoir bag. With lower tidal volume, ie, 4-8 $\mathrm{ml} / \mathrm{kg}$ predicted body weight and lower inspiratory pressure, mechanical ventilation should be performed. It's given for sepsis induce respiratory failure patients as well. $6 \mathrm{ml} / \mathrm{kilogram}$ tidal PBW is the initial tidal volume which can range to $8 \mathrm{ml} /$ kilogram on the counter of undesirable side effects. The $\mathrm{pH}$ goal of 7.3 to7.45 must be achieved for permitting hypercapnia. Target tidal volumes should be achieved with the help of deep sedation to control respiratory drive (Zhao H, et al 2017).

16 to 18 hours of mechanical ventilation is required in patients with severe ARDS in a day which ultimately requires sufficient human resources and expertise. High PEEP is suggested instead of low PEEP for patients with moderate or severe ARDS. With access to the known individuals of extracorporeal life support in the settings, despite of ventilation by protection of lung should be considered with reference to refractory hypoxemia patients. Authorised centres should provide adequate case volume for maintaining the expertise wrt, ECLS and can require COVID 19 application of IPC measures (Gawai,et al 2020). In the disconnection of ventilator there is loss of PEEP and results in atelectasis which should be avoided. In line catheters are helpful for clamp ET tube and airway suctioning, if requirement is disconnection.

\section{Low Cost Made In India Ventilators Boon Or Bane?:} When the pandemic hit the country and hospitals increased the alarming need for ventilators, which numbered 47,000, the government came forward with the idea of going local for the manufacturing of low-cost ventilators. Since imports were expensive and difficult due to global travel restrictions, companies like AgVa Healthcare and state-owned Bharat Electronics ltd/ BEL were given orders of 10,000 to 20,000 units each. In the month of May, 16 local businesses were awarded contracts to produce 60,000 cost-effective ventilators in two months. The PM CARES fund was used to buy these computers, which totalled 20 billion rupees. The highend ventilators that doctors used previously cost 1.5 to 2 million rupees each, while the AgVa ventilators cost 150,000 rupees each and BEL's machines cost 400,000 rupees each (Iyengar K et al 2020). 
Manufacturers sped up production and reduced costs by ignoring more sophisticated features due to the short timeframe. After receiving 175 ventilators $(155$ from BEL and 20 from AgVa), the LokNayak Jai Prakash Hospital, Delhi's largest for covid 19 treatment, said last week that the machines did not have BiPAP, a primary non-invasive choice for supplying oxygen without intubation.

To avoid harm from the invasive procedure in the early stages, several senior consultants recommended using non-invasive techniques first in mild to moderate cases before the patient is on the verge of not being able to breathe on their own. According to the current situation in India, most patients do not need invasive ventilation and are treated with non-invasive techniques only before their condition worsens (Burhani,et al 2020). A ventilator without control button for the oxygen level and pressure could harm a patient seemingly low-end and are totally unacceptable. 81 machines were returned back to $\mathrm{AgVa}$ Manufacturers with the opinion to not stand the critical care of Covid patients. A report from Mumbai doctors sent to medical education and research suggested that AgVa machines were incapable of reaching the targeted oxygen saturation and indicate the actual level of FiO2 delivered to the patient. Patients showed signs of improvement when shifted to other ventilators who were showing the signs of restlessness and exhaustion with local ventilators.

Chances are aerosolisation at a very high intensity in BiPAP by the mask through which Covid patient gets oxygen, spreading it to the medical staff taking care of them in the modern scenario of Air conditioned with no windows Intensive care units, in the form of droplets from the holes present in mask. This ultimately raised a question regarding the usage of which type of ventilators best suitable both for the patients as well as the medical staff and if Indian government overestimated the country's need for them, as with the evolvement of pandemic more doctors are using non-invasive technique for treatment. Data reported by a Mumbai based consultant pulmonologists states that, on the deterioration of corona infected patient the lungs are compliant even when the body think it needs oxygen, then the only requirement is to fulfil the demand for oxygen and let patient breathe better, further, intubation is only required if the patient arrives late with Acute Respiratory Distress Syndrome.

Overall most critical care medical professionals insisted on the presence of both non- invasive and invasive recourse of the care of Covid-19 patients. BEL and AgVa representatives are speaking with hospitals concerned and trying to explore the upgraded versions of the same (Prasad, Narayan et al 2020, Wanjari, A. K., et al 2020 and Kolhe, et al 2020). Earlier due to this alarming pandemic condition which then stated the shortage of ventilators, has now turned into trouble due to the overabundance of manufacturing of more than 60,000 ventilators, turned out not so reliable for the current situation by the doctors. Winded Indian manufacturers now hope that foreign markets with shortages will buy their ventilators.
With the concluding statement based on database from 23rd June 2020, showcased by the Health Ministry if India, asserted, only 4.16 percentile of infected Covid patients required ventilators where as the need for oxygen stood a 16 percentage.

\section{CONCLUSION}

As a result, disease prevention at the earliest stage is considered a condemnatory measure. However, one must be prepared for any mishaps in the manifestation of disease as it progresses to complication. Invasive procedures such as mechanical ventilation and noninvasive procedures such as nasal cannula oxygenation must be used successfully, depending on the patient's capacity for reaction to the care offered. Given the unpredictability of the future in relation to the disease, it would be advantageous if the country were to be equipped in advance with manufactured low-cost ventilators.

\section{REFERENCES}

Burhani, T.S. and Naqvi, W.M., 2020. Telehealth--A Boon in the Time of COVID 19 Outbreak. Journal of Evolution of Medical and Dental Sciences, 9(29), pp.2081-2085.

Gawai, J.P., Singh, S., Taksande, V.D., Sebastian, T., Kasturkar, P. and Ankar, R.S., 2020. Critical Review on Impact of COVID 19 and Mental Health.

Iyengar, K., Bahl, S., Vaishya, R. and Vaish, A., 2020. Challenges and solutions in meeting up the urgent requirement of ventilators for COVID-19 patients. Diabetes \& Metabolic Syndrome: Clinical Research \& Reviews, 14(4), pp.499-501.

Jha, R.K., Jain, P. and Ambad, R.S., 2020. The origin, transmission and mortality of corona virus. Journal of critical reviews, 7(10), pp.93-96.

Latchoumi, T.P., Ezhilarasi, T.P. and Balamurugan, K., 2019. Bio-inspired weighed quantum particle swarm optimization and smooth support vector machine ensembles for identification of abnormalities in medical data. SN Applied Sciences, 1(10), pp.1-10.

Lentz, S., Roginski, M.A., Montrief, T., Ramzy, M., Gottlieb, M. and Long, B., 2020. Initial emergency department mechanical ventilation strategies for COVID-19 hypoxemic respiratory failure and ARDS. The American Journal of Emergency Medicine.

Lu, H., Stratton, C.W. and Tang, Y.W., 2020. Outbreak of pneumonia of unknown etiology in Wuhan, China: the mystery and the miracle. Journal of medical virology, 92(4), pp.401-402.

Naqvi, W.M. and Sahu, A., 2020. Paradigmatic Shift in the Education System in a Time of COVID 19. Journal of Evolution of Medical and Dental Sciences, 9(27), pp.1974-1977.

Prasad, N., Bhatt, M., Agarwal, S.K., Kohli, H.S., 
Gopalakrishnan, N., Fernando, E., Sahay, M., Rajapurkar, M., Chowdhary, A.R., Rathi, M. and Jeloka, T., 2020. The adverse effect of COVID pandemic on the care of patients with kidney diseases in India. Kidney international reports, 5(9), pp.1545-1550..

Sison, S.M., Sivakumar, G.K., Caufield-Noll, C., Greenough III, W.B., Oh, E.S. and Galiatsatos, P., 2021. Mortality outcomes of patients on chronic mechanical ventilation in different care settings: A systematic review. Heliyon, 7(2), p.e06230.

Thakur, K., Kumar, N. and Sharma, N., 2020. Effect of the pandemic and lockdown on mental health of children.
The Indian Journal of Pediatrics, 87, pp.552-552. Wanjari, A.K., Dubey, A., Chaturvedi, S. and Kumar, S., 2020. Young COVID 19 presenting as fatal subarachnoid hemorrhage: Association or chance. Medical Science, 24(104), pp.2712-2715.

Zhao, H., Wang, H., Sun, F., Lyu, S. and An, Y., 2017. High-flow nasal cannula oxygen therapy is superior to conventional oxygen therapy but not to noninvasive mechanical ventilation on intubation rate: a systematic review and meta-analysis. Critical care, 21(1), pp.112. 